\title{
Variations in Hepatic Angiosis and Their Significance in Hepatic Pathology
}

\author{
Margarita Theodorakidou, Olti-Alexandra Nikola, George Ioannis Lambrou* \\ First Department of Pediatrics, Choremeio Research Laboratory, Hematology/Oncology Unit, National and Kapodistrian University of \\ Athens, Athens, Greece \\ Email address: \\ mtheodorakidou@gmail.com (Margarita T.), alexandra.ni28@hotmail.com (Olti-Alexandra N.), glamprou@med.uoa.gr (George I. L.) \\ ${ }^{*}$ Corresponding author
}

\section{To cite this article:}

Margarita Theodorakidou, Olti-Alexandra Nikola, George Ioannis Lambrou. Variations in Hepatic Angiosis and Their Significance in Hepatic Pathology. International Journal of Clinical and Developmental Anatomy. Vol. 3, No. 5, 2017, pp. 36-44.

doi: 10.11648/j.ijcda.20170305.11

Received: October 29, 2017; Accepted: December 6, 2017; Published: January 8, 2018

\begin{abstract}
The knowledge of liver blood vessels anatomy is essential for hepatic transplants and hepatobiliary and pancreatic procedures. In addition, it is crucial to be aware of the liver anatomy due to its importance in the operations of the hepatic neoplasms. Studies have shown that hepatic perfusion presents a number of variants, which have an important role in liver physiology as well as in the treatment of hepatoblastic neoplasms. The purpose of the present work is the presentation and analysis of anatomic variations and anomalies of the hepatic artery, and the importance and application of this knowledge in clinical practice of liver diseases. In particular, the significance of this insight into hepatoblastic neoplasms is presented.
\end{abstract}

Keywords: Angiosis, Hepatic Arteries, Hepatic Veins, Hepatic Neoplasms

\section{Introduction}

The liver is a vital organ of the vertebrates (Figure 1). It presents a wide spectrum of actions for instance detoxification, protein synthesis and the production of biochemical substances necessary for food digestion. The liver is an essential organ for life preservation hence; the ways to compensate its functions so far are limited to short term liver dialysis in case of its absence. The organ possesses a fundamental role in metabolism and a majority of functions including glucagon storage, erythrocyte destruction and plasma protein synthesis, production of hormones and excretion of endogenous or exogenous toxic substances. It is located under the diaphragm in the upper right quadrant, the right hypochondrium and reaches the middle upper part of the abdomen, the epigastrium. The liver is responsible for bile production; an alkaline mixture which degrades simple and complex molecules required for several physiological features of the organism.

The dual hepatic blood supply is provided by the portal vein in percentage of $75 \%$ and the rest $25 \%$ by the hepatic artery. The portal vein transfers the venous blood from the spleen and the gastro- intestinal tract with its individual organs to the liver, while the hepatic artery transfers arterial blood to the same destination as well. The demands for oxygen are covered equally by both suppliers. The blood passes through the capillaries into the central vein of each lobule. The central venules are merged to liver veins, which abandon the liver.

The knowledge of liver anatomy is essential for hepatic transplants and hepatobiliary and pancreatic procedures. In the predominant pattern, found in $50-80 \%$ of the population, the common hepatic artery, after its insertion from the abdominal artery, is divided into branches, including the hepatic artery in particular, divided into right and left. The international literature has discussed multiple variants of this model. Michels first presented and classified these variants with his colleagues in 1966 [1]. Following the study of 200 anatomical preparations, they proposed 10 different models of the hepatic artery, with more frequent presence or substitution of the adjuvant left or right hepatic artery. This international classification was modified in 1994 by Hiatt and his associates. After the study of 1000 patients, Hiatt reduced the models from ten to five basics and to a sixth, more infrequent. The most common variants according to Hiatt are the right hepatic artery sprout from the upper mesenteric 
artery and the left hepatic artery outcome from the left gastric artery [2]. Ectopic is defined as the hepatic artery located in a position rather unusual. Adjuvant is characterized as the extra hepatic artery that may be additional to the normal one. An adjuvant hepatic artery is usually smaller than the primary, but it can be just as functional and important. An ectopic or adjuvant hepatic artery may bleed part of a hepatic lobe usually an adjunctive hepatic artery - or even the entire lobe. Knowing the existence of such an artery is of great importance because its ligation can lead to extensive necrosis of the liver parenchyma.

The aim of this essay is to present and analyze the anatomic variants and abnormalities of the hepatic artery, as well as the significance and application of this knowledge in modern surgical procedures of the liver.

\section{Brief Anatomy and Physiology of the Liver (Functions)}

The liver is a brownish red organ and it can be divided in four lobes of different sizes and shapes. The normal weight of the human liver is between 1.4-1.6 kg (3.1-3.5 lb) and accounts for $2 \%$ of the total body weight. It occupies totally the right hypochondrium, part of the epigastrium and reaches the left hypochondrium.
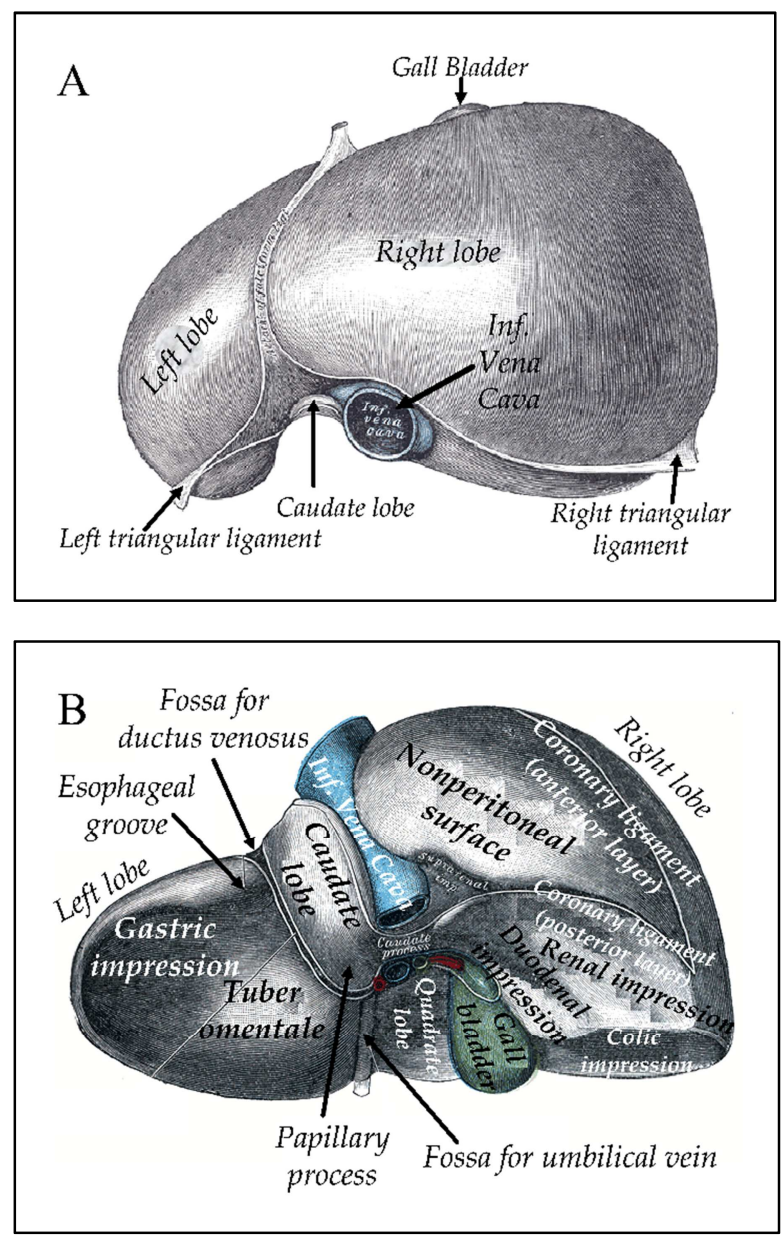
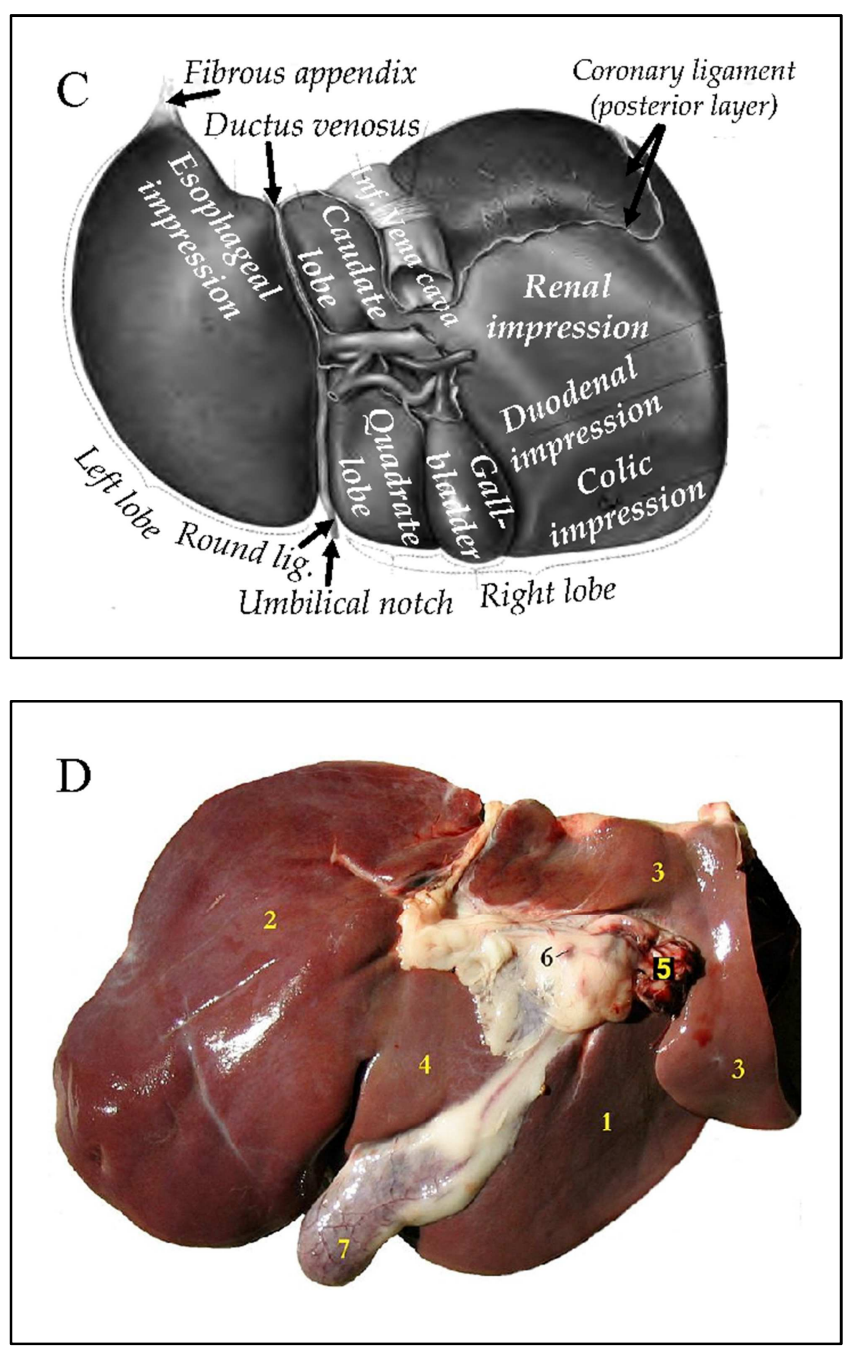

Figure 1. Schematic liver representations. The front side of the left and right lobes (source of the image by: Henry Gray's Anatomy, Plate 1085) ${ }^{I}$ (Henry Vandyke Carter-Henry Gray (1918)), the rear side with the basic arteries image by: Henry Vandyke Carter-Henry Gray 1918. Anatomy of the Human Body: Gray's Anatomy, Plate 1087) 2 (B), the rear side with the four lobes (source of the image by: Dr. Johannes Sobotta-Sobotta's Atlas and Text (2) the left lobe, (3) Lobus caudatus, (4) the lobus quadratus hepatis, (1) the lobus quadratus hepatis 5) the hepatic artery, (6) the hepatic glands and (7) gall bladder (D) (source: https://en.wikipedia.org/wiki/Liver).

It is wedged shaped with the base facing the right lateral side and the apex towards the left. The liver possesses 3 borders (anterior, left and right) and 3 surfaces (superior, inferior and posterior). Porta hepatis (hilum) is located on the inferior surface of the liver and contains the common hepatic duct, the hepatic artery and the portal vein. The hepatic artery supplies the liver with oxygenated blood, while the portal vein carries rich in nutrients blood from the gastrointestinal tract, the spleen and the pancreas to the liver. These vessels are divided into capillaries and each one ends in a lobule. Each lobule is formed by million hepatocytes, the functional cells of the liver. The inferior liver surface contains the fossa for the gallbladder and various impressions formed by the pressure of the adjacent vicera.

1 Material under Creative Commons License. 


\subsection{Biliary System}

The biliary system is also known as biliary tree hence to the tree like bifurcations of the biliary tracts. Bile is produced by the liver and collected by the bile ductules, which merge to form the bile ducts. Inside the liver parenchyma these ducts are called intrahepatic bile ducts while the part outside of the liver is called extrahepatic bile tract. The intrahepatic ducts finally form the left and right hepatic duct, which merge to form the common hepatic duct. The junction of the cystic duct from the gallbladder with the common hepatic duct creates the common bile duct with the pancreatic duct form the ampulla of Vater in the second part of the jejunum. As a result, the bile can be secreted directly into the jejunum through the common bile duct, or can be temporarily stored in the gallbladder via the cystic duct.

\subsection{Peritoneal Liganments}

Except for a small part of the superior surface of the liver, under the diaphragm, uncovered, the rest of the liver surface is covered by the peritoneum, a thin double layered serous membrane which prevents friction with the adjacent viscera. The peritoneum is folded and forms the falciform ligament and the left and right triangular ligaments. These ligaments have no functional uses and are not related with the joint ligaments. Lacking of any functional use and not related to the joint ligaments, they can be used as anatomical landmarks. An exception is the falciform ligament which attaches the liver to the posterior surface of the anterior abdominal wall.

\subsection{Lobes}

Traditionally, the liver is divided by surface landmarks into four lobes. Falciform ligament divides the superior surface into the right and left lobes. The elevation of the liver exposes the posterior surface and two more lobes are revealed between the left and right lobe. These are the cuadate and quadrate lobes. In the posterior surface they are divided by the ligamentum venosum and the round ligament of the liver (on the left there is the left lobe). The transverse fissure (porta hepatis) separates the caudate from the quadrate lobe and the coronary ligament with the fossa for the vena cava separates the right lobe from the caudate and quadrate lobe. Each lobe consists of hepatic lobules. The hepatic lobules have a central vein that drains to the hepatic vein which transfers blood outside the liver. The periphery of the hepatic lobules contains bile ducts, arteries, veins and lymphatics transferring substances to and from the hepatic lobules.

\subsection{Functional Anatomy}

In a central part of the liver called liver hilum or porta hepatis, the portal vein and the common hepatic artery enter in the liver and the common bile duct exits. These two vessels and the common bile duct are divided in to left and right branches. Each branch supplies the left and right functional lobes of the liver. These two functional (true) lobes can be divided by an imaginary plane that runs from the fossa of the gallbladder to the fossa of the inferior vena cava. The middle hepatic vein also divides the true lobes. The right hepatic vein separates the right lobe in to an anterior and to a posterior segment and the left lobe is further divided to a medial and lateral segment by the left hepatic vein. The middle segment is called quadrate lobe. The widely used classification system by Coinaud divides the liver in eight functional segments as presented in Table 1 and Figure 2 respectively. The caudate lobe is considered to be a separate structure that receives blood from both left and right branches of the portal and hepatic vessels.

Table 1. Correspondence between anatomical lobes and Couinaud segments.

\begin{tabular}{ll}
\hline Section* & Sections according to Couinaud \\
\hline Caudal & 1 \\
Lateral & 2,3 \\
Medium & $4 \mathrm{a}, 4 \mathrm{~b}$ \\
Right & $5,6,7,8$ \\
\hline * or lobe, in the case of the caudal lobe \\
Each number in the list corresponds to a table number. \\
1. Caudal \\
2. Upper lateral section \\
3. Lower lateral section \\
4. a. Upper medium section \\
$\quad$ b. Lower medium section \\
5. Lower rear section \\
6. Lower frontal section \\
7. Upper frontal section \\
8. Upper rear section
\end{tabular}

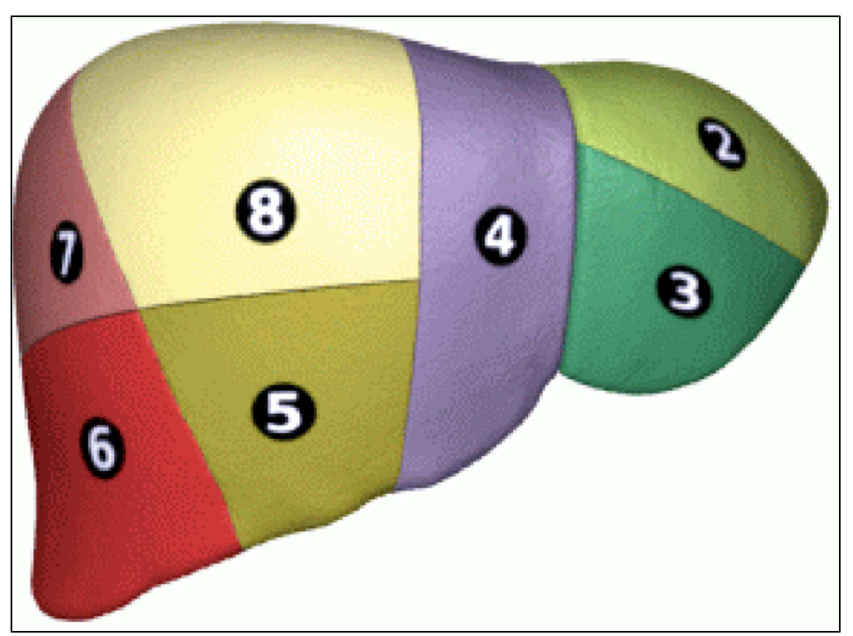

Figure 2. Schematic representation of a liver classified by Couinaud. The eight parts of the liver, divided by their functional structure, are presented. The main characteristic of this classification is the independent perfusion of each functional structure (source: https://en.wikipedia.org/wiki/Liver) ${ }^{2}$.

\subsection{Physiology}

Hepatocytes are responsible for the various functions of the liver. At present, there is not any artificial organ or device available that can substitute for the whole array of liver functions. An experimental therapy for liver failure is called

2 Material under Creative Commons License. 
liver dialysis and can be the substitute for some of the liver functions. The liver has various roles in carbohydrate metabolism: glyconeogenesis (glycose synthesis from certain amino acids, lactic acid or glycerol), glycogenolysis (the breakdown of glycogen to glucose) and glycogenesis (glycogen synthesis from glucose). Liver has a pivotal role in protein metabolism (synthesis and degradation). Furthermore, the liver contributes to lipid metabolism: cholesterol synthesis, lipogenesis (production of triglycerides). Blood coagulation factors are also synthesized in the liver: I (fibrinogen), II (prothrombin), V, VII, IX, X, $\mathrm{XI}$, protein $\mathrm{C}$, protein $\mathrm{S}$, and antithrombin.

During the first trimester of the fetal life the liver is the main site of erythrocyte production. After the $32^{\text {th }}$ week the bone marrow is responsible for erythropoiesis. Liver secretes bile, a (yellowish) fluid that emulsifies lipids. A part of the bile is directly secreted into the jejunum, while the rest is temporarily stored in the gallbladder. Liver is the site of synthesis of the insulin like growth factor 1 (IGF-1), a polypeptide hormone important for the childhood growth and in adults has anabolic actions. The liver produces a major part of thrombopoietin; a glycoprotein hormone that regulates platelet production from the bone marrow.

\subsection{Other Liver Functions}

The liver stores a variety of substances including glucose (in the form of glycogen), vitamin A (supply for 1-2 years), vitamin D (for 1-4 months), vitamin B12 (for 1-3 years), iron and copper. It also contributes to the immune system by its reticuloendothelial system which consists of a variety of immune competent cells that act like a filter to the antigens transported by the portal circulation. Albumin is produced in the liver and is a plasma protein which helps in the regulation of oncotic pressure in blood. Angiotensinogen produced by the liver is a hormone that increases blood pressure when is activated by renin (an enzyme produced by the kidneys in response to the lowering of blood pressure).

\section{Variations in Hepatic Perfusion}

The profile of hepatic perfusion is known to be variable. Changes in the way the liver receives the total amount of blood from the hepatic junction of the celiac artery takes place in $25 \%$ to $75 \%$ of the cases [3]. In other cases, however, the lobes may be perfused by the upper mesenteric artery, the left gastric artery, or collateral branches. These vessels may be complementary, thus, they may be present as additional deposits in normal blood supply or as replacements of primary arterial supply of lobes. In the study of this anatomical phenomenon, a substantial tool is liver transplantation. The arteries leading to the liver should be conscientiously studied during the liver removal process by the donor, aiming at avoidance of causing injuries and inadequate perfusion of the liver in the recipient. This finding has received great attention from researchers in recent years, since the profile of liver perfusion is obviously of great importance [4]. Therefore, the profound knowledge of the location of all the arteries is essential. The findings of 1000 patients observed and studied by Hiatt et al. (1994) presented such differences in hepatic perfusion [2]. The observations of Michels (1966) and Hiatt (1994) demonstrated differences in the classification of the ways of hepatic perfusion with the second giving a simpler typology. The following tables summarize the results of their study (Table 2, Table 3 and Table 4). The variation between the types of hepatic perfusion differs from study to study, indicating how complex hepatic anatomy is. The types of perfusion reported (Type 1-5, other) refer to the following types of perfusion:

a) Type $1(n=757)$ : refers to the physiological profile where the hepatic artery originates from the celiac artery forming the gastrointestinal artery. The hepatic artery is divided into two branches, the left and the right.

b) Type $2(n=97)$ : The adjuvant artery is derived from the left gastric artery.

c) Type $3(n=106)$ : The adjuvant right hepatic artery originates from the upper mesenteric artery.

d) Type $4(n=23)$ : The right hepatic artery originates from the upper mesenteric artery and the left hepatic artery is a branch of the left gastric artery.

Type $5(n=15)$ : the entire hepatic artery is derived from a branch of the upper mesenteric artery.

e) Type 6 (other) $(n=2)$ : The normal hepatic artery originates directly from the aorta.

f) The results of the comparative study are shown diagrammatically in Figure 3 , in addition to the anatomical types of perfusion, as reported by Hiatt et al. (1994) [5].

Table 2. Classification of Michels hepatic arteries [1].

\begin{tabular}{lll}
\hline TYPE & DESCRIPTION & PERCENTAGE (\%) \\
\hline 1 & NATURAL INSERTION & 55 \\
2 & ECTOPIC LEFT HEPATIC ARTERY FROM LEFT GASTRIC & 10 \\
5 & ADJUVANT LEFT HEPATIC ARTERY & 8 \\
3 & ECTOPIC RIGHT HEPATIC ARTERY FROM SUPERIOR MESENTERIC & 11 \\
6 & ADJUVANT RIGHT HEPATIC ARTERY & 7 \\
4 & ECTOPIC RIGHT AND LEFT HEPATIC ARTERY & 1 \\
7 & ADJUVANT RIGHT AND LEFT HEPATIC ARTERY & 2 \\
8 & ECTOPIC RIGHT HEPATIC ARTERY+ADJUVANT LEFT HEPATIC ARTERY OR ECTOPIC LEFT & 2,5 \\
9 & HEPATIC ARTERY+ADJUVANT RIGHT HEPATIC ARTERY & 0,5 \\
\hline 10 & COMMON HEPATIC ATERY FROM SUPERIOR MESENTERIC & 1 \\
\hline
\end{tabular}


Table 3. Classification of hepatic artery types in a Michels and Hiatt comparative study. Adjusted and reproduced from Hiatt et al. (1994) [2].

\begin{tabular}{llll}
\hline Publication & Type & Description & (\%) \\
\hline & 1 & Normal & 55 \\
& 2 & Replaced Left Hepatic Artery (LHA) from Left Gastric Artery (LGA) & 10 \\
& 5 & Accessory LHA & 8 \\
Michels (n=200) & 3 & Replaced Right Hepatic Artery (RHA) from Superior Mesenteric Artery (SMA) & 18 \\
& 6 & Accessory RHA & 7 \\
& 4 & Replaced RHA \& LHA & 18 \\
& 7 & Accessory RHA \& LHA & 1 \\
& 8 & Replaced RHA \& Accessory LHA or Replaced LHA \& Accessory RHA & 1 \\
& & & 2 \\
& 9 & Common Hepatic Artery (CHA) from SMA & 4 \\
& 10 & CHA from LGA & 2.5 \\
& 1 & Normal & 0.5 \\
& 2 & Replaced or Accessory LHA & 75.5 \\
& 3 & Replaced or Accessory RHA & 9.7 \\
& 4 & Replaced or Accessory RHA + & 10.6 \\
& 5 & Replaced or Accessory RHA & 2.3 \\
& 6 & CHA from SMA & 1.5 \\
\end{tabular}

LHA-left hepatic artery; LGA-left gastric artery; RHA-right hepatic artery; SMA-superior mesenteric artery; CHA-common hepatic artery.

Table 4. Comparative Study of Hepatic Blood Pressure. Adjusted and reproduced from Hiatt et al. (1994) [2].

\begin{tabular}{|c|c|c|c|c|c|c|c|}
\hline \multirow{2}{*}{ Type } & Hiatt [2] & Michels [1] & Rong [6] & Kemeny [7] & Rygaard $[8]$ & Daly [9] & Niederhuber [10] \\
\hline & $(n=1000)$ & $(n=200)$ & $(n=120)$ & $(n=100)$ & $(n=216)$ & $(n=200)$ & $(n=111)$ \\
\hline 1 & 75.7 & 55 & 51 & $59 *$ & 75.5 & 76 & 73 \\
\hline 2 & 9.7 & 18 & 12 & 17 & 4.6 & 7.7 & 10 \\
\hline 3 & 10.6 & 18 & 21 & 18 & & 12 & 11 \\
\hline 4 & 2.3 & 4 & & 2 & 1.9 & & 2 \\
\hline 5 & 1.5 & 2.5 & 5 & 3 & 1.4 & & \\
\hline Other & 0.2 & 0.5 & 11 & 1 & 3.2 & 6 & 5 \\
\hline
\end{tabular}

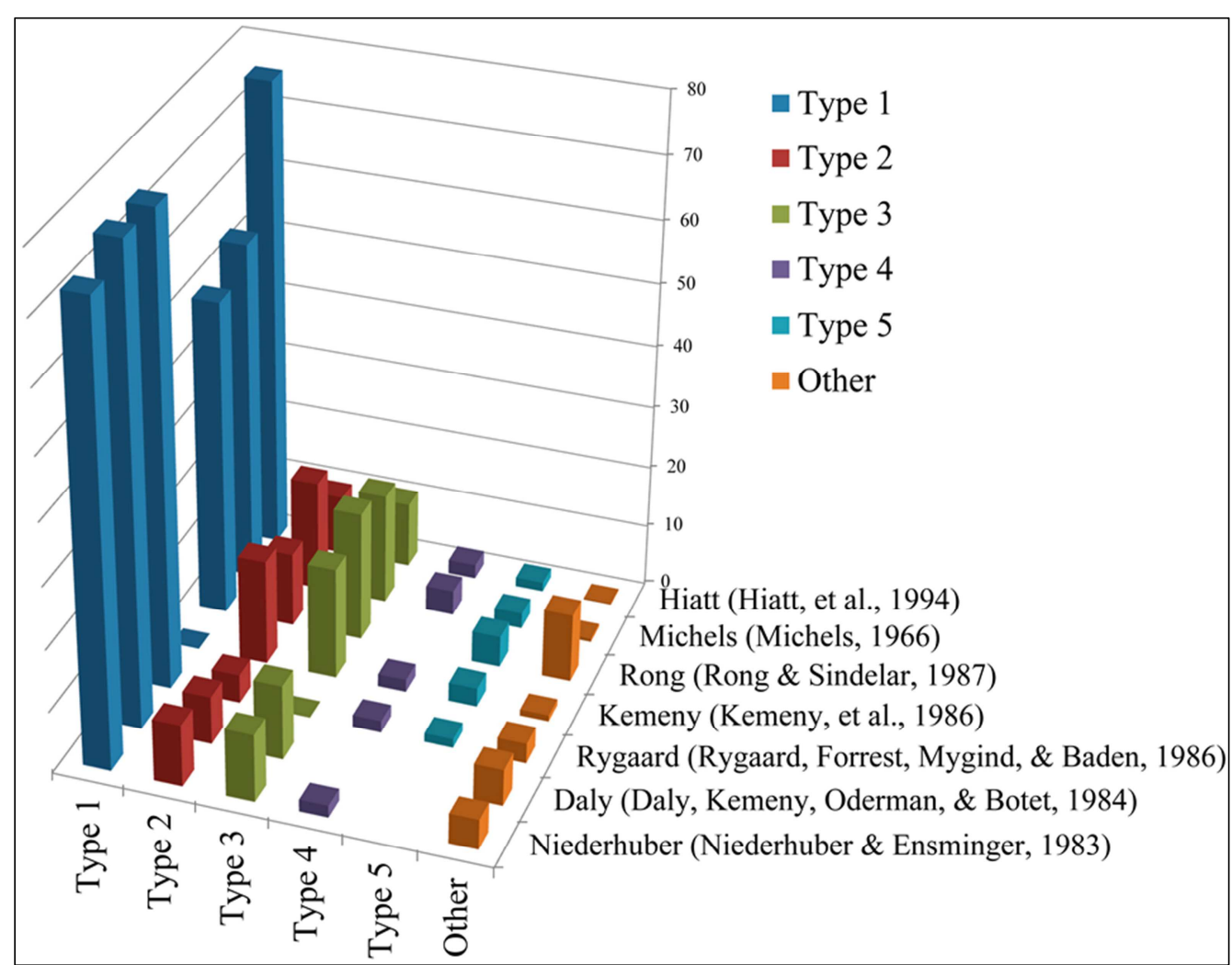

Figure 3. Diagram of the percentages of hepatic perfusion types in the studies reported therein. The chart was reproduced based on the data of Hiatt et al (1994) [2]. 


\section{Clinical Implications of Variant Hepatic Perfusion in Hepatic Pathology}

The liver supports almost every organ of the body and is necessary for life. Due to its positioning strategy and multidimensional functions, the liver is also vulnerable to numerous ailments. The most common diseases are: infections such as hepatitis A, hepatitis B, C, E, alcoholic disease, fatty liver, cirrhosis, cancer, pharmaceutical failure (especially acetaminophen, also known as paracetamol and anticancer medicines). A great number of liver diseases are accompanied by jaundice, caused by elevated levels of bilirubin. Bilirubin is formed during the degradation of aged red blood cell hemoglobin. Normally, the liver removes the bilirubin from the circulation via excretion of bile. The main pediatric liver diseases are biliary atresia, $\alpha 1$-antitrypsin deficiency, Alagille's syndrome, progressive familial endothelial cholestasis and Langerhans cell-site haemocytosis.

Diseases interfering with liver function lead to disruption of physiological processes. However, the liver has increased capacity of regeneration and redundancy, therefore, in most cases, symptoms occur only after extensive hepatic parenchyma destruction. Liver diseases are diagnosed by liver function tests, such as the measurement of the enzymes SGOT (glutamic oxaloacetic transaminase) and SGPT (glutamic pyruvic transaminase), $\gamma$-GT $(\gamma$ glutamyltransferase), LDH (lactate dinitrogenase), direct and indirect plasma bilirubin, as well as the production of acute phase proteins.

The liver is the only internal organ of the human body that has the ability to physically regenerate a missing tissue. Only $25 \%$ of the hepatic tissue is sufficient to regenerate the whole liver. Nevertheless, this regeneration is rather compensation. Removed lobes do not grow back, and liver regeneration involves restoring its function rather than its normal structure, in contrast to true regeneration, where both functionality and anatomy are restored.

Hepatocytes have a major part in this process by reentering into the cell cycle; they migrate from the inactive $G_{0}$ phase of the cycle to the $G_{I}$ phase and undergo mitosis. The process is activated by $\mathrm{p} 75$ receptors. Indications for bivalent stem cells, called precursor liver cells, are believed to be in Hering's canals. These cells can be differentiated either in hepatocytes or in cholangiocytes (the cells that surface the bile ducts).

Scientific and medical studies on the regenerative capacity of the liver often refer to the Greek Titan Prometheus, who, according to the myth, was chained to a rock on the Mount Caucasus, where an eagle devoured his liver on a daily basis, but the organ was regenerated during the night. Some believe that the myth reveals the awareness of ancient Greeks of the liver regeneracy, but this view is disputed.

Thus, knowledge of the liver arteries has an extremely important role in clinical practice. An example of this critical knowledge is liver transplantation, such as Split Liver Transplantation (SLT), in which two recipients receive a transplant from a single donor. Differentiation of the hepatic arteries should be probed meticulously to affirm the success of the procedure [11]. Similar recent reports highlight the importance of the hepatic arteries knowledge in rebuilding the arteries during liver transplantation [12].

Respectively, this knowledge is extended to the diagnosis of liver hematoma [13]. Likewise, the significance of vasculature is critical for surgical procedures; removal of the pancreas or small intestine. Reports indicate cases, during surgical procedures, where the surgeon could not decide ad hoc whether an artery is ectopic or normal, resulting in an incorrect incision and consequently, necrosis of liver sections [14].

In the discipline of oncology, hepatic arteries are really valuable. Hepatocellular carcinoma (HCC) and metastatic liver neoplasms constitute the majority of liver tumors. The prognosis of both remains poor, a challenge for research and a clinical interest of various medical specialists who are assigned to diagnose and treat liver neoplasms. Apart from the neoplasias characterized to be in limited extent and can be surgically resected, giving a better prognosis, a great deal of cases will be considered inoperable, either forthwith at diagnosis or in the course of a known disease. The factors which differentiate resectable from unresectable liver damages include; the size of the tumor, the extent of hepatic parenchyma occupied, and the location of tumors within the liver parenchyma, the histological type, the multiplicity of tumor sites, the possible infiltration of the liver portal vein, or even rarely, of other vascular branches, for instance diaphragm and liver capsule, the degree of vascularization of the tumor, the possibility of liver chemoembolization. In most inoperable hepatic neoplasms, primary or secondary, part of the alternative treatment protocol may be performed by Invasive Radiology. In contemporary hospital units, the Invasive Radiologist is tasked with the implementation of an alternative treatment regimen by methods of chemoembolization in order to treat the disease, inhibit its progression and extend the survival rate. Trans-catheter arterial chemoembolization of the liver (TACE) was introduced in the mid-1970s. With the exception of unresectable hepatic tumors, TACE can be used as a preoperative or postoperative part of the treatment. Preceded the surgical intervention, the method can alter the staging of the tumor and the potential of resection. In either case, preceded or followed by hepatectomy, TACE reduces the likelihood of postoperative recurrence of the tumor as well as the risk of developing intrahepatic metastases of a preexisting $\mathrm{HCC}$, thereby prolonging survival. Although the prognosis of metastatic liver disease is usually poor, the increasing use of aggressive treatment regimens in some patients (such as patients with metastatic neuroendocrine tumors and metastases of colon cancer) can lead to a favorable outcome. The effectiveness of TACE method is based on 3 parameters. 
a) The blood supply of the liver is unique. The portal vein provides $75 \%$ of the liver blood supply, while $25 \%$ comes from the hepatic artery. This backup blood supply allows the embolization of branches of the hepatic artery without subsequent hepatic necrosis.

b) $95 \%$ of the blood and the primary and metastatic liver tumors originate from the hepatic artery.

c) In the early $1980 \mathrm{~s}$, studies revealed that oily iodinated contrast agent (lipiodol) injected into the hepatic artery preferably remains in the neoplastic arterial network of existing malignant proliferation. For this purpose, the anatomy of the patient has to be completely understood in order to allow liver catheterization and proper administration of chemotherapeutics in most cases [15]. Hepatic artery angiography can be used for neoplasms of the bile duct that cause outward compression of the hepatic vessels. Such interventions make the knowledge of blood perfusion and anatomy of the liver mandatory for the planning of surgical procedure [16].

\section{Neoplasms Accompanied by Vascular Lesions}

\subsection{Pancreatoblastoma}

Pancreatoblastoma is a rare malignant pancreatic neoplasm of the childhood. Since first described in 1957, more than 200 cases have been reported. Reviews in literature suggest that even if pancreatoblastoma is considered to be malignant, it presents better prognosis in the absence of metastasis than the adult pancreatic malignant tumors. The study of the biology of this rare tumor aims to define the prognosis and to optimize the therapeutic strategies [17].

Pancreatoblastoma is usually diagnosed in children under the age of 10 years old. The immune phenotype is determined by the predominant component of the tumor such as the acinar, ductal, squamous corpuscles, and endocrine components. The localization of the tumor is mainly the pancreatic head and it has the tendency to invade in adjacent organs and vessels. For diagnosis and staging, MRI, CT, and abdominal ultrasonography can be performed. The measurement of $\alpha$ - fetoprotein (AFP) and of lactic dehydrogenase (LDH) can be useful. Treatment relies mainly on surgical resection with adjuvant chemotherapy. Radiation therapy has fewer implications in the treatment of pancreatoblastoma [18].

\subsection{Undifferentiated Embryonal Sarcoma of the Liver as a Cause of Spontaneous Liver Rupture}

The undifferentiated embryonal sarcoma of the liver is another rare malignant neoplasm of the children. Stocker and Ishak introduced the term in 1978 but the etiology of the undifferentiated embryonal sarcoma is still obscure because of its rarity [19]. Authors suggest that the undifferentiated embryonal sarcomas arise from the malignant transformation of a benign mesenchymal hamartoma, while other groups hypothesize a link to the existence of another malignancy such as fibro sarcoma, leiomyosarcoma, liposarcoma, rhabdomyosarcoma, or hepatocellular carcinoma. The finding that the undifferentiated embryonal sarcoma is not accompanied by cirrhosis as in the $29 \%$ of cases in other primary liver sarcomas, make the latter theory more unfavorable. For some liver neoplasms, the etiology is the mutation of hepatic stem cell, but additional immunohistochemical methods performed to clarify the pathogenesis of the undifferentiated embryonal sarcoma created more confusion.

The absence of specific early symptoms and the rapid growth of the tumor make clinical diagnosis difficult and, at the time of diagnosis, the patients were found in an advanced stage. Imaging techniques can be employed for diagnosis and staging. The radiologic image is of large smooth masses with clear border, giving the impression of hematomas. For definite diagnosis, histological examination may be required.

Even if the data are not sufficient, the goal of treatment is local resection of the tumor. Liver parenchyma is not cirrhotic hence the success of resection can be as high as $70 \%$. There is not enough evidence to support an improvement in prognosis after liver transplantation.

To date, a standard chemotherapeutic regimen is not accomplished and multiple drug combinations have been tested. In children with tumor recurrence, cytogenetic techniques revealed translocation between regions of chromosome 7 and 11 combined with expression of multidrug resistance genes (mdrl genes) which lead to reduction of the therapeutic effects of chemotherapy.

When the surgical resection of the tumor is not complete, postoperative radiotherapy is performed. After the completion of chemotherapy or radiation therapy is completed a second look laparotomy is performed in order to exclude local recurrence. Prognosis is better in children compared to adult patients and knowledge of chromosomal ploidy with the use of flow cytometry seems to be of great value in determining prognosis.

In rare cases of spontaneous liver rupture, differential diagnosis should include the undifferentiated embryonal sarcoma in order to perform resection of the tumor [20].

\subsection{Intrahepatic Vascular Shunts During Childhood Development}

Hepatic vascular aneurysms may be considered to be a consequence of hepatic injuries or intrahepatic tumors. However, childhood developmental malformations, which could cause fatal complications, have rarely been reported. In a study in 1997, clinical and radiological analysis was performed in 24 patients. The abnormalities identified were related to: direct communication to the hepatic artery, congenital arteriovenous malformations of the hepatic artery and portal vein disorders, and congenital splenorenal anastomosis with permanent flow through the venous duct. Despite the rarity of the dysfunctions, immediate treatment with surgical and radiological methods is a necessity. The methods could identify and characterize liver damage, quantify perfusion, 
control portal vein anatomy and resilience, map the organ for vascular ligation and embolization, and identify possible lesions in other organs [21].

\subsection{Polysplenic Syndrome}

Polyspleny is a rare form of congenital malformation. Most patients do not reach adulthood due to the heart abnormalities associated with this syndrome. Vascular abnormalities of the veins are also common, mainly of the inferior vena cava and of the azygous- hemiazygous vein. Arterial abnormalities are even rare, occurring during childhood and involving an anatomic divergence, where the primary hepatic artery is originated from the superior mesenteric artery, or an arteriovenous lung aneurysm. In Polyspleny, a splenic fissure is reported and rare as well [22].

\subsection{Diagnostic Approach and Treatment}

"Scintigraphy" is the technique where images of the hepatic pathway are performed by technetium- $99 \mathrm{~m}$ injection (Tc-99m or $99 \mathrm{mTc}$ ), a radioactive isotope emitting gamma rays and having a half-life of 6 hours. Following the technique, the static scintigraphy of the liver is conducted. These tests have made it possible to differentiate vascularized liver tumors such as hepatoma, hepatoblastoma, hepatic adenoma, cavernous hemangioma from post-vascular lesions such as metastatic diseases and abscesses. The scintigraphy method emits a small dose of radiation but requires appropriate equipment and skilled personnel [23].

\subsection{Use of Interferon in Case of Hepatic Hemangioma}

Hepatic hemangioendothelioma may be fatal for children, especially when typical methods of treatment have failed. However, treatment with interferon $\alpha$ can have positive impact. Specifically, a study in 2000 referred to a case of a 14-month-old child, where interferon $\alpha$ administration led to regression of heart failure, calcification and decreased blood flow, leading to the conclusion that interferon- $\alpha$ therapy contributes to the control of the course of the disease [24].

\subsection{Modern Therapeutic Strategies}

Surgery has been the main therapeutic approach for treating hepatoblastoma so far. Various systems are used for staging and prognosis of the disease, with PRETEXT being prevalent. The majority of cases with hepatoblastoma undergo preoperative chemotherapy, which affords numerous variations and is of the utmost importance in choosing the optimal way of surgery. Patients with PRETEXT IV grade tumors, multifocal tumors, and aggressive tumors in liver vessels are often driven to liver transplantation, a process that requires great attention and absolute specialization. Pulmonary tumor metastasis gives a poor prognosis, however, when promptly diagnosed, pulmonary nodules can be effectively controlled by chemotherapy or partial pneumonectomy, allowing the corollary of transplantation. The TACE method, as aforementioned, despite to date relatively few reports, is a useful tool in the management of hepatoblastoma. However, TACE is considered to be the most prevalent, especially among methods proven ineffective or inappropriate. The most common side effects are; increased body temperature, abdominal pain, nausea, increase of transaminase and liver enzymes, while less frequent complications include acute liver failure, liver infarction, liver abscess, tumor rupture and pulmonary embolism. The technique demands meticulous handling, full awareness of its advantages and disadvantages and qualified in invasive radiology personnel [25].

\subsection{MRI Imaging Approaches and Clinical Features of Benign Hyper Vascular Hepatic Nodules in Childhood Cancer Survivors}

Benign hepatic nodules with rich perfusion in survivors of childhood cancer are presented after high-dose chemotherapy and hematopoietic stem cell transplantation. The imaging characteristics of MRI include intense arterial enhancement and lack of washout during the delayed phase. Benign nodules, usually numerous and small in size, are formed several years after chemotherapy or marrow transplantation. Advanced MRI methods contribute to the differentiation of hepatic nodules in patients and can perform a diagnostic role in focal nodular hyperplasia (FNH) [26].

\section{Conclusions}

Knowledge of hepatic vasculature and its variations is an accepted fact of great significance as the planning and implementation of superior abdominal surgery requires high knowledge of liver anatomy and its variants. The applications of this knowledge range from simple liver interventions to even complicated issues; transplantation and chemoembolization.

\section{Disclosures and Conflict of Interest}

The authors have nothing to disclose and no conflict of interest.

\section{References}

[1] N. A. Michels, "Newer anatomy of the liver and its variant blood supply and collateral circulation," The American Journal of Surgery, vol. 112, pp. 337-347, 1966.

[2] J. R. Hiatt, J. Gabbay, and R. W. Busuttil, "Surgical anatomy of the hepatic arteries in 1000 cases," Annals of surgery, vol. 220, p. 50, 1994.

[3] P. H. Lin and E. L. Chaikof, "Embryology, anatomy, and surgical exposure of the great abdominal vessels," Surgical Clinics of North America, vol. 80, pp. 417-433, 2000.

[4] K. Ishigami, Y. Zhang, S. Rayhill, D. Katz, and A. Stolpen, "Does variant hepatic artery anatomy in a liver transplant recipient increase the risk of hepatic artery complications after transplantation?," American Journal of Roentgenology, vol. 183, pp. 1577-1584, 2004 
[5] J. R. Hiatt, J. Gabbay, and R. W. Busuttil, "Surgical anatomy of the hepatic arteries in 1000 cases," Ann Surg, vol. 220, pp. 50-2, Jul 1994.

[6] G. Rong and W. Sindelar, "Aberrant peripancreatic arterial anatomy. Considerations in performing pancreatectomy for malignant neoplasms," The American surgeon, vol. 53, pp. 726-729, 1987.

[7] M. M. Kemeny, J. M. Hogan, D. A. Goldberg, C. Lieu, J. D. Beatty, W. A. Kokal, et al., "Continuous hepatic artery infusion with an implantable pump: problems with hepatic artery anomalies," Surgery, vol. 99, pp. 501-504, 1986.

[8] H. Rygaard, M. Forrest, T. Mygind, and H. Baden, "Anatomic variants of the hepatic arteries," Acta Radiologica. Diagnosis, vol. 27, pp. 425-427, 1986.

[9] J. M. Daly, N. Kemeny, P. Oderman, and J. Botet, "Long-term hepatic arterial infusion chemotherapy: anatomic considerations, operative technique, and treatment morbidity," Archives of Surgery, vol. 119, pp. 936-941, 1984.

[10] J. E. Niederhuber and W. D. Ensminger, "Surgical considerations in the management of hepatic neoplasia," in Seminars in oncology, 1983, pp. 135-147.

[11] E. Chaib, M. Ribeiro, W. A. Saad, and J. Gama-Rodrigues, "The main hepatic anatomic variations for the purpose of split-liver transplantation," in Transplantation proceedings, 2005, pp. 1063-1066.

[12] M. Takatsuki, Y.-C. Chiang, T.-S. Lin, C.-C. Wang, A. Concejero, C.-C. Lin, et al., "Anatomical and technical aspects of hepatic artery reconstruction in living donor liver transplantation," Surgery, vol. 140, pp. 824-828, 2006.

[13] M. A. Konstam, R. A. Novelline, and C. A. Athanasoulis, "Aberrant hepatic artery: a potential cause for error in the angiographic diagnosis of traumatic liver hematoma," Abdominal Imaging, vol. 4, pp. 43-45, 1979.

[14] J. A. Adamthwaite, N. Pennington, and K. V. Menon, "Anomalous hepatic arterial anatomy discovered during pancreaticoduodenectomy," Surgical and Radiologic Anatomy, vol. 29, pp. 269-271, 2007.

[15] K.-S. Jeng and H.-J. Ching, "The role of surgery in the management of unusual complications of transcatheter arterial embolization for hepatocellular carcinoma," World journal of surgery, vol. 12 , pp. 362-367, 1988.
[16] K. Miyashita, K. Shiraki, T. Ito, H. Taoka, and T. Nakano, "The right hepatic artery syndrome," World Journal of Gastroenterology: WJG, vol. 11, p. 3008, 2005.

[17] L. Sheng, Z. Weixia, Y. Longhai, and Y. Jinming, "Clinical and biologic analysis of pancreatoblastoma," Pancreas, vol. 30, pp. 87-90, 2005.

[18] M. W. Saif, "Pancreatoblastoma," JOP: Journal of the pancreas, vol. 8, pp. 55-63, 2007.

[19] J. T. Stocker and K. G. Ishak, "Undifferentiated (embryonal) sarcoma of the liver. Report of 31 cases," Cancer, vol. 42, pp. 336-348, 1978.

[20] S. Yedibela, T. Reck, R. Ott, V. Müller, T. Papadopoulos, and W. Hohenberger, "Undifferentiated, embryonal sarcoma as a rare cause of spontaneous liver rupture in adults," Der Chirurg; Zeitschrift fur alle Gebiete der operativen Medizen, vol. 71, pp. 101-105, 2000.

[21] M. Paley, P. Farrant, P. Kane, N. Heaton, E. Howard, and J. Karani, "Developmental intrahepatic shunts of childhood: radiological features and management," European radiology, vol. 7, pp. 1377-1382, 1997.

[22] T. Ergun, H. Lakadamyali, H. Lakadamyali, and O. Eldem, "Adult polysplenic syndrome accompanied by aberrant right subclavian artery and hemangioma in a cleft spleen: a case report," Annals of vascular surgery, vol. 22, pp. 579-581, 2008 .

[23] G. F. Gates, J. H. Miller, and P. Stanley, "Scintiangiography of hepatic masses in childhood," JAMA, vol. 239, pp. 26672670,1978

[24] B. Le Luyer, A. Duquenoy, J. Poinsot, J. Boulloche, G. Gaussin, and P. Le Roux, "Use of interferon in a case of hepatic hemangioma," Archives de pediatrie: organe officiel de la Societe francaise de pediatrie, vol. 7, pp. 1201-1204, 2000 .

[25] T. Hishiki, "Current therapeutic strategies for childhood hepatic tumors: surgical and interventional treatments for hepatoblastoma," International journal of clinical oncology, vol. 18, pp. 962-968, 2013.

[26] S.-Y. Yoo, J. H. Kim, H. Eo, T. Y. Jeon, K. W. Sung, and H. S. Kim, "Dynamic MRI findings and clinical features of benign hypervascular hepatic nodules in childhood-cancer survivors," American Journal of Roentgenology, vol. 201, pp. 178-184, 2013. 